\title{
SHARP TWO-WEIGHT, WEAK-TYPE NORM INEQUALITIES FOR SINGULAR INTEGRAL OPERATORS
}

\author{
D. Cruz-Uribe, SFO and C. PÉrez
}

Abstract. We give a sufficient condition for singular integral operators and, more generally, Calderón-Zygmund operators to satisfy the weak $(p, p)$ inequality

$$
u\left(\left\{x \in \mathbb{R}^{n}:|T f(x)|>t\right\}\right) \leq \frac{C}{t^{p}} \int_{\mathbb{R}^{n}}|f|^{p} v d x, \quad 1<p<\infty .
$$

Our condition is an $A_{p}$-type condition in the scale of Orlicz spaces:

$$
\|u\|_{L(\log L)^{p-1+\delta, Q}}\left(\frac{1}{|Q|} \int_{Q} v^{-p^{\prime} / p} d x\right)^{p / p^{\prime}} \leq K<\infty, \quad \delta>0 .
$$

This conditions is stronger than the $A_{p}$ condition and is sharp since it fails when $\delta=0$.

\section{Introduction}

Let $T$ be a Calderón-Zygmund singular integral operator:

$$
T f(x)=\text { p.v. } \int_{\mathbb{R}^{n}} K(x-y) f(y) d y,
$$

where the kernel $K$ is continuously differentiable on $\mathbb{R}^{n} \backslash\{0\}$, has zero average on the unit sphere, and for all $x \neq 0$,

$$
|K(x)| \leq \frac{C}{|x|^{n}} \quad \text { and } \quad|\nabla K(x)| \leq \frac{C}{|x|^{n+1}} .
$$

More generally, we may assume that $T$ is a Calderón-Zygmund operator. For a precise definition, see Coifman and Meyer [4], Journé [9] or Christ [3]. In this paper we consider the problem of finding sufficient conditions on a pair of weights $(u, v)$ - i.e., non-negative, locally integrable functions - such that $T$ satisfies the weak $(p, p)$ inequality $(1<p<\infty)$ :

$$
u\left(\left\{x \in \mathbb{R}^{n}:|T f(x)|>t\right\}\right) \leq \frac{C}{t^{p}} \int_{\mathbb{R}^{n}}|f|^{p} v d x, \quad t>0, \quad f \in L^{p}(v) .
$$

Received March 17, 1999.

1991 Mathematics Subject Classification. 42B20, 42B25

Key words and prhases. weights, singular integral operators, Calderón-Zygmund operators, maximal operators, Orlicz spaces

The first author is supported by a Ford Foundation fellowship; the second author by DGICYT Grant PB40192, Spain. 
In the one-weight case - i.e., $u=v$ - a sufficient condition is $v \in A_{p}$, and this condition is necessary if $n=1$ and $T$ is the Hilbert transform. (For sufficiency, see Journé [9]; for necessity, see Hunt, Muckenhoupt and Wheeden [8].) In the two-weight case, however, $(u, v) \in A_{p}$ is no longer sufficient: see Muckenhoupt and Wheeden [12] or section 4 below for a counter-example. Recall that $(u, v) \in A_{p}$ if there exists a positive constant $K$ such that for all cubes $Q$,

$$
\left(\frac{1}{|Q|} \int_{Q} u d x\right)\left(\frac{1}{|Q|} \int_{Q} v^{-p^{\prime} / p} d x\right)^{p / p^{\prime}} \leq K .
$$

Neugebauer [13] showed that if there exists $r>1$ such that $\left(u^{r}, v^{r}\right) \in A_{p}$ then (1.1) holds, and in fact $T$ is strong $(p, p)$. In [5] we showed that if we strengthened the $A_{p}$ condition by only adding a "power bump" to the left-hand term, then we obtained a sufficient condition for $T$ to be weak $(p, p)$.

Theorem 1.1. Let $T$ be a Calderón-Zygmund operator. Given a pair of weights $(u, v)$ and $p, 1<p<\infty$, suppose that for some $r>1$ and for all cubes $Q$,

$$
\left(\frac{1}{|Q|} \int_{Q} u^{r} d x\right)^{1 / r}\left(\frac{1}{|Q|} \int_{Q} v^{-p^{\prime} / p} d x\right)^{p / p^{\prime}} \leq K<\infty .
$$

Then inequality (1.1) holds.

In this paper we improve Theorem 1.1 by replacing the power bump in (1.2) by a smaller "Orlicz bump". Given a Young function $B$ and a cube $Q$, the mean Luxemburg norm of a measurable function $f$ on $Q$ is defined by

$$
\|f\|_{B, Q}=\inf \left\{\lambda>0: \frac{1}{|Q|} \int_{Q} B\left(\frac{|f|}{\lambda}\right) d x \leq 1\right\} .
$$

When $B(t)=t \log (1+t)^{p-1+\delta}$, this norm is also denoted by $\|\cdot\|_{L(\log L)^{p-1+\delta}, Q}$. (More information on Orlicz spaces is in Section 2 below.) Our main result is the following.

Theorem 1.2. Let $T$ be a Calderón-Zygmund operator. Given a pair of weights $(u, v)$ and $p, 1<p<\infty$, suppose that for some $\delta>0$ and for all cubes $Q$,

$$
\|u\|_{L(\log L)^{p-1+\delta, Q}}\left(\frac{1}{|Q|} \int_{Q} v^{-p^{\prime} / p} d x\right)^{p / p^{\prime}} \leq K<\infty .
$$

Then for every $t>0$ and $f \in L^{p}(v)$,

$$
u\left(\left\{x \in \mathbb{R}^{n}:|T f(x)|>t\right\}\right) \leq \frac{C}{t^{p}} \int_{\mathbb{R}^{n}}|f|^{p} v d x .
$$

Further, this result is sharp since it does not hold in general when $\delta=0$. 
Remark 1.3. Theorem 1.2 is stronger than the result proved in [5], but our proof only works for Calderón-Zygmund operators. We do not know if the corresponding results proved in [5] for fractional integrals and commutators are true with power bumps replaced by Orlicz bumps.

Remark 1.4. As a corollary to Theorem 1.2 we get a new proof of the one-weight, strong $(p, p)$ norm inequality for Calderón-Zygmund operators. If $w \in A_{p}$ then both $w$ and $w^{-p^{\prime} / p}$ satisfy the reverse Hölder inequality, so for some $\epsilon>0$, (1.4) holds with $p$ replaced by $p \pm \epsilon$. The strong-type inequality then follows by interpolation.

Remark 1.5. An analogue of Theorem 1.2 for the two-weight, strong $(p, p)$ inequality for the Hilbert transform on the unit circle was proved by Treil, Volberg and Zheng [21]. However, their method does not extend to Calderón-Zygmund operators.

The proof of Theorem 1.2 proceeds as follows: given a Young function $A$, define the maximal function

$$
M_{A} f(x)=\sup _{Q \ni x}\|f\|_{A, Q} .
$$

In [16] it was shown that if $A(t)=t \log (1+t)^{\epsilon}, \epsilon>0$, and $T$ is any CalderónZygmund operator, then for any weight $w$ and function $f$,

$$
w\left(\left\{x \in \mathbb{R}^{n}:|T f(x)|>t\right\}\right) \leq \frac{C_{\epsilon}}{t} \int_{\mathbb{R}^{n}}|f| M_{A} w d x
$$

We combine this inequality with a duality argument to reduce the proof to characterizing the weights $(u, v)$ such that $M_{A}$ is bounded from $L^{p^{\prime}}\left(u^{-p^{\prime} / p}\right)$ to $L^{p^{\prime}}\left(v^{-p^{\prime} / p}\right)$. We do this by extending the techniques used in [18].

We note that independently and much earlier, Muckenhoupt and Wheeden [11] conjectured that inequality (1.6) holds when $T$ is the Hilbert transform and $M_{A}$ is replaced by the Hardy-Littlewood maximal operator. Assuming this, they proved that inequality (1.5) holds for the Hilbert transform and for pairs $(u, v)$ such that $\left(u^{-p^{\prime} / p}, v^{-p^{\prime} / p}\right)$ satisfy the Sawyer condition $S_{p^{\prime}}$. (They also conjectured that this condition is necessary.) Their proof uses a duality argument which is essentially the same as ours.

If the Muckenhoupt-Wheeden conjecture is true for Calderón-Zygmund operators, then our proof of Theorem 1.2 yields the stronger result that inequality (1.5) holds if in inequality (1.4) we replace the Orlicz norm $\|\cdot\|_{L(\log L)^{p-1+\delta, Q}}$ by the weaker Orlicz norm $\|\cdot\|_{B, Q}$, where $B$ is a doubling Young function which satisfies the growth condition

$$
\int_{c}^{\infty}\left(\frac{t}{B(t)}\right)^{p^{\prime}-1} \frac{d t}{t}<\infty
$$


For example, we can take $B(t) \approx t \log (t) \log \log (t)^{p-1+\delta}, \delta>0$. (See Section 2 for the relevance of this condition.) Irrespective of the validity of the MuckenhouptWheeden conjecture, we believe this result is true.

The remainder of this paper is organized as follows: in Section 2 we gather a few results about Orlicz spaces (some of which we alluded to above) that we will use in the proofs. In Section 3 we prove a sufficient, $A_{p}$-type condition in the scale of Orlicz spaces for the strong $(p, p)$ norm inequality for $M_{A}$. Since we can do so with no additional work, we prove the result for a larger class of Young functions $A$ than is necessary to prove Theorem 1.2. Finally, in Section 4 we prove Theorem 1.2. Our proof actually gives a slightly stronger result; we state it precisely at the end of the section.

Throughout this paper all notation is standard or will be defined as needed. All cubes are assumed to have their sides parallel to the co-ordinate axes. Given a cube $Q, l(Q)$ will denote the length of its sides and for any $r>0, r Q$ will denote the cube with the same center as $Q$ and such that $l(r Q)=r l(Q)$. By weights we will always mean non-negative, locally integrable functions which are positive on a set of positive measure. Given a Lebesgue measurable set $E$ and a weight $w,|E|$ will denote the Lebesgue measure of $E$ and $w(E)=\int_{E} w d x$. Given $1<p<\infty, p^{\prime}=p /(p-1)$ will denote the conjugate exponent of $p$. Finally, $C$ will denote a positive constant whose value may change at each appearance.

\section{Preliminary results on Orlicz spaces}

In this section we summarize a few facts about Orlicz spaces. (For more information, see Bennett and Sharpley [1] or Rao and Ren [19].) A function $B:[0, \infty) \rightarrow[0, \infty)$ is a doubling Young function if it is continuous, convex and increasing, if $B(0)=0$ and $B(t) \rightarrow \infty$ as $t \rightarrow \infty$, and if it satisfies $B(2 t) \leq$ $C B(t)$ for all $t>0$. For Orlicz norms we are usually only concerned about the behavior of Young functions for $t$ large. Given two functions $B$ and $C$, we write $B(t) \approx C(t)$ if $B(t) / C(t)$ is bounded and bounded below for $t \geq c>0$.

Recall that we defined the localized Luxembourg norm by equation (1.3); an equivalent norm which is often useful in calculations is due to Krasnosel'skil and Rutickiı [10, p. 92] (also see Rao and Ren [19, p. 69]):

$$
\|f\|_{A, Q} \leq \inf _{\mu>0}\left\{\mu+\frac{\mu}{|Q|} \int_{Q} A\left(\frac{|f|}{\mu}\right) d x\right\} \leq 2\|f\|_{A, Q} .
$$

A Young function $B$ is said to satisfy the $B_{p}$ condition if for some constant $c>0$,

$$
\int_{c}^{\infty} \frac{B(t)}{t^{p}} \frac{d t}{t} \approx \int_{c}^{\infty}\left(\frac{t^{p^{\prime}}}{\bar{B}(t)}\right)^{p-1} \frac{d t}{t}<\infty .
$$

(Cf., condition (1.7) above.) Here $\bar{B}$ is the complementary Young function associated to $B$; it has the property that for all $t>0$,

$$
t \leq B^{-1}(t) \bar{B}^{-1}(t) \leq 2 t .
$$


In [18] it was shown that if $B$ is doubling then $B \in B_{p}$ if and only if $M_{B}: L^{p} \rightarrow$ $L^{p}$ is bounded. Examples of functions satisfying the $B_{p}$ condition are $B(t)=t^{q}$, $1 \leq q<p$. More interesting examples are given by

$$
B(t)=t^{p} \log (1+t)^{-(1+\delta)} \quad B(t) \approx t^{p} \log (t)^{-1} \log \log (t)^{-(1+\delta)}, \quad \delta>0 .
$$

Finally, we will need a generalization of Hölder's inequality due to O'Neil [14]. (Also see Rao and Ren [19, p. 64].)

Lemma 2.1. Let $A, B$ and $C$ be Young functions such that for all $t>0$,

$$
B^{-1}(t) C^{-1}(t) \leq A^{-1}(t) .
$$

Then for all functions $f$ and $g$ and all cubes $Q$,

$$
\|f g\|_{A, Q} \leq 2\|f\|_{B, Q}\|g\|_{C, Q}
$$

\section{Strong-type norm inequalities for $M_{A}$}

In this section we prove the following weighted norm inequality.

Theorem 3.1. Given $p, 1<p<\infty$, let $A, B$, and $C$ be Young functions such that $B^{-1}(t) C^{-1}(t) \leq A^{-1}(t), t>0$, and $C$ is doubling and satisfies the $B_{p}$ condition. If $(u, v)$ is a pair of weights such that for every cube $Q$,

$$
\left(\frac{1}{|Q|} \int_{Q} u d x\right)^{1 / p}\left\|v^{-1 / p}\right\|_{B, Q} \leq K<\infty
$$

then for every function $f \in L^{p}(v)$,

$$
\int_{\mathbb{R}^{n}}\left(M_{A} f\right)^{p} u d x \leq C \int_{\mathbb{R}^{n}}|f|^{p} v d x
$$

Remark 3.2. Since $B^{-1}(t) \rightarrow \infty$ as $t \rightarrow \infty, C^{-1}(t) \leq A^{-1}(t)$ for all $t$ sufficiently large. Hence $A(t) \leq C(t)$ for $t$ large and so $A \in B_{p}$. As we noted above, when $u=v=1$ this condition is also necessary.

We first prove a lemma which generalizes the Calderón-Zygmund decomposition.

Lemma 3.3. Given a Young function $A$, suppose $f$ is a non-negative function such that $\|f\|_{A, Q}$ tends to zero as $l(Q)$ tends to infinity. Given a $>2^{n+1}$, for each $k \in \mathbb{Z}$ there exists a disjoint collection of maximal dyadic cubes $\left\{Q_{j}^{k}\right\}$ such that for each $j$,

$$
a^{k}<\|f\|_{A, Q_{j}^{k}} \leq 2^{n} a^{k}
$$

and

$$
\left\{x \in \mathbb{R}^{n}: M_{A} f(x)>4^{n} a^{k}\right\} \subset \bigcup_{j} 3 Q_{j}^{k} .
$$


Further, let $D_{k}=\bigcup_{j} Q_{j}^{k}$ and $E_{j}^{k}=Q_{j}^{k} \backslash\left(Q_{j}^{k} \cap D_{k+1}\right)$. Then the $E_{j}^{k}$ 's are pairwise disjoint for all $j$ and $k$ and there exists a constant $\alpha>1$, depending only on a, such that $\left|Q_{j}^{k}\right| \leq \alpha\left|E_{j}^{k}\right|$.

Remark 3.4. To have $f$ satisfy the hypotheses of Lemma 3.3, it suffices to assume that $f$ is bounded and has compact support.

Proof. The existence of dyadic cubes $\left\{Q_{j}^{k}\right\}$ with the desired properties is shown in [18]. In the case $A(t)=t$ the existence of $\alpha$ is proved in [17] (cf., GarciaCuerva and Rubio de Francia [7, p. 398]). Here we extend that proof to general A.

Since the $Q_{j}^{k}$ 's are disjoint for fixed $k$, by their maximality the $E_{j}^{k}$ 's are pairwise disjoint for all $j$ and $k$. Further, by inequality (3.2),

$$
\begin{aligned}
\left|Q_{j}^{k} \cap D_{k+1}\right| & =\sum_{i}\left|Q_{j}^{k} \cap Q_{i}^{k+1}\right| \\
& =\sum_{i: Q_{i}^{k+1} \subset Q_{j}^{k}}\left|Q_{i}^{k+1}\right| \\
& \leq a^{-k-1} \sum_{i: Q_{i}^{k+1} \subset Q_{j}^{k}}\left|Q_{i}^{k+1}\right|\|f\|_{A, Q_{i}^{k+1}},
\end{aligned}
$$

by inequality $(2.1)$,

$$
\begin{aligned}
& \leq a^{-k-1} \sum_{i: Q_{i}^{k+1} \subset Q_{j}^{k}}\left|Q_{i}^{k+1}\right| \inf _{\mu>0}\left\{\mu+\frac{\mu}{\left|Q_{i}^{k+1}\right|} \int_{Q_{i}^{k+1}} A\left(\frac{|f|}{\mu}\right) d x\right\} \\
& =a^{-k-1} \sum_{i: Q_{i}^{k+1} \subset Q_{j}^{k}} \inf _{\mu>0}\left\{\mu\left|Q_{i}^{k+1}\right|+\mu \int_{Q_{i}^{k+1}} A\left(\frac{|f|}{\mu}\right) d x\right\} \\
& \leq a^{-k-1} \inf _{\mu>0}\left\{\mu\left|Q_{j}^{k} \cap D_{k+1}\right|+\mu \int_{Q_{j}^{k} \cap D_{k+1}} A\left(\frac{|f|}{\mu}\right) d x\right\} \\
& \leq a^{-k-1} \inf _{\mu>0}\left\{\mu\left|Q_{j}^{k}\right|+\mu \int_{Q_{j}^{k}} A\left(\frac{|f|}{\mu}\right) d x\right\} \\
& \leq 2 a^{-k-1}\left|Q_{j}^{k}\right|\|f\|_{A, Q_{j}^{k}},
\end{aligned}
$$

again by inequality $(3.2)$,

$$
\leq 2^{n+1} a^{-1}\left|Q_{j}^{k}\right|
$$

It follows immediately from this that

$$
\left|E_{j}^{k}\right| \geq\left|Q_{j}^{k}\right|-2^{n+1} a^{-1}\left|Q_{j}^{k}\right|
$$

which establishes the desired inequality with $\alpha=\left(1-2^{n+1} a^{-1}\right)^{-1}$. 
Proof of Theorem 3.1. Fix a function $f \in L^{p}(v)$. Without loss of generality we may assume that $f$ is non-negative; by a standard approximation argument we may also assume that $f$ is bounded and has compact support.

Fix $a>2^{n+1}$; for $k \in \mathbb{Z}$ let

$$
\Omega_{k}=\left\{x \in \mathbb{R}^{n}: 4^{n} a^{k}<M_{A} f(x) \leq 4^{n} a^{k+1}\right\} .
$$

Then by Lemma 3.3,

$$
\Omega_{k} \subset \bigcup_{j} 3 Q_{j}^{k}, \quad \text { where } \quad\|f\|_{A, Q_{j}^{k}}>a^{k} .
$$

We can therefore estimate as follows:

$$
\begin{aligned}
\int_{\mathbb{R}^{n}}\left(M_{A} f\right)^{p} u d x & =\sum_{k} \int_{\Omega_{k}}\left(M_{A} f\right)^{p} u d x \\
& \leq C \sum_{k} a^{k p} u\left(\Omega_{k}\right) \\
& \leq C \sum_{j, k} a^{k p} u\left(3 Q_{j}^{k}\right) \\
& \leq C \sum_{j, k} u\left(3 Q_{j}^{k}\right)\|f\|_{A, Q_{j}^{k}}^{p},
\end{aligned}
$$

by Lemma 2.1,

$$
\begin{aligned}
& =C \sum_{j, k} u\left(3 Q_{j}^{k}\right)\left\|f v^{1 / p} v^{-1 / p}\right\|_{A, Q_{j}^{k}}^{p} \\
& \leq C \sum_{j, k} u\left(3 Q_{j}^{k}\right)\left\|v^{-1 / p}\right\|_{B, Q_{j}^{k}}^{p}\left\|f v^{1 / p}\right\|_{C, Q_{j}^{k}}^{p} .
\end{aligned}
$$

It follows from (1.3) that $\left\|v^{-1 / p}\right\|_{B, Q_{j}^{k}} \leq 3^{n}\left\|v^{-1 / p}\right\|_{B, 3 Q_{j}^{k}}$. Hence, by Lemma 3.3 and condition (3.1), and since the $E_{j}^{k}$, s are disjoint,

$$
\begin{aligned}
& \leq C \sum_{j, k}\left(\frac{1}{\left|3 Q_{j}^{k}\right|} \int_{3 Q_{j}^{k}} u d x\right)\left\|v^{-1 / p}\right\|_{B, 3 Q_{j}^{k}}^{p}\left\|f v^{1 / p}\right\|_{C, Q_{j}^{k}}^{p}\left|E_{j}^{k}\right| \\
& \leq \sum_{j, k} \int_{E_{j}^{k}} M_{C}\left(f v^{1 / p}\right)^{p} d x \\
& \leq C \int_{\mathbb{R}^{n}} M_{C}\left(f v^{1 / p}\right)^{p} d x \\
& \leq C \int_{\mathbb{R}^{n}} f^{p} v d x
\end{aligned}
$$

The last inequality holds since $C$ is doubling and in $B_{p}$, and so as we noted in Section $2, M_{C}$ is bounded on $L^{p}$. This completes the proof. 


\section{Proof of Theorem 1.2}

In this section we prove Theorem 1.2. Fix $p, 1<p<\infty$, and $\delta>0$. Let $A(t)=t \log (1+t)^{\epsilon}$, where $0<\epsilon<\delta / p$. Let $\eta=\delta-\epsilon p$. Then

$$
\begin{aligned}
A^{-1}(t) & \approx \frac{t}{\log (1+t)^{\epsilon}} \\
& =\frac{t^{1 / p}}{\log (1+t)^{\epsilon+(p-1+\eta) / p}} \times t^{1 / p^{\prime}} \log (1+t)^{(p-1+\eta) / p} \\
& =B^{-1}(t) C^{-1}(t),
\end{aligned}
$$

where

$$
B(t) \approx t^{p} \log (1+t)^{(1+\epsilon) p-1+\eta}=t^{p} \log (1+t)^{p-1+\delta}
$$

and

$$
C(t) \approx t^{p^{\prime}} \log (1+t)^{-1-\left(p^{\prime}-1\right) \eta} .
$$

(This triple of Young functions is originally due to O'Neil [15].) It follows at once from the definition that $C$ is doubling and satisfies the $B_{p^{\prime}}$ condition. Therefore, by Theorem 3.1, $M_{A}: L^{p^{\prime}}\left(u^{-p^{\prime} / p}\right) \rightarrow L^{p^{\prime}}\left(v^{-p^{\prime} / p}\right)$ is bounded, provided that

$$
\left(\frac{1}{|Q|} \int_{Q} v^{-p^{\prime} / p} d x\right)^{1 / p^{\prime}}\left\|u^{1 / p}\right\|_{B, Q} \leq K<\infty .
$$

But this is equivalent to condition (1.4), since by a change of variables,

$$
\left\|u^{1 / p}\right\|_{B, Q} \approx\|u\|_{L(\log L)^{p-1+\delta}}^{1 / p} .
$$

Now fix $f$. By a standard approximation argument we may assume without loss of generality that $f \in C^{\infty}\left(\mathbb{R}^{n}\right)$ and has compact support.

For each $t>0$, let $\Omega_{t}=\left\{x \in \mathbb{R}^{n}:|T f(x)|>t\right\}$. This set is bounded, so $u\left(\Omega_{t}\right)<\infty$. By duality, there exists a non-negative function $G \in L^{p^{\prime}},\|G\|_{p^{\prime}}=1$, such that

$$
u\left(\Omega_{t}\right)^{1 / p}=\left\|u^{1 / p} \chi_{\Omega_{t}}\right\|_{p}=\int_{\Omega_{t}} u^{1 / p} G d x
$$

by inequality (1.6),

$$
\leq \frac{C}{t} \int_{\mathbb{R}^{n}}|f| M_{A}\left(u^{1 / p} G\right) d x
$$

by Hölder's inequality,

$$
\begin{aligned}
& =\frac{C}{t} \int_{\mathbb{R}^{n}}|f| v^{1 / p} v^{-1 / p} M_{A}\left(u^{1 / p} G\right) d x \\
& \leq C\left(\frac{1}{t^{p}} \int_{\mathbb{R}^{n}}|f|^{p} v d x\right)^{1 / p}\left(\int_{\mathbb{R}^{n}} M_{A}\left(u^{1 / p} G\right)^{p^{\prime}} v^{-p^{\prime} / p} d x\right)^{1 / p^{\prime}} .
\end{aligned}
$$


By the boundedness of $M_{A}$ noted above, and since $\|G\|_{p^{\prime}}=1$,

$$
\begin{aligned}
& \leq C\left(\frac{1}{t^{p}} \int_{\mathbb{R}^{n}}|f|^{p} v d x\right)^{1 / p}\left(\int_{\mathbb{R}^{n}} G^{p^{\prime}} d x\right)^{1 / p^{\prime}} \\
& =C\left(\frac{1}{t^{p}} \int_{\mathbb{R}^{n}}|f|^{p} v d x\right)^{1 / p} .
\end{aligned}
$$

If we raise both sides of the final inequality to the power $p$ we get inequality (1.5).

To show that the hypotheses of Theorem 1.2 are sharp, consider the following example on the real line due to Muckenhoupt [11]. Fix $p=2$; it follows from an inequality due to Stein [20] that for any function $u, M_{L(\log L)} u \approx M^{2} u$. (Also see Carozza and Passarelli [2].) Further, for any cube $Q$,

$$
\begin{aligned}
& \|u\|_{L(\log L), Q} \cdot \frac{1}{|Q|} \int_{Q} M_{L(\log L)} u(x)^{-1} d x \leq \\
& \frac{1}{|Q|} \int_{Q} M_{L(\log L)} u(x) M_{L(\log L)} u(x)^{-1} d x \leq 1,
\end{aligned}
$$

so the pair $\left(u, M_{L(\log L)} u\right)$ satisfies inequality (1.4) with $\delta=0$. If we let

$$
u(x)=(x \log x \log \log x)^{-1} \chi_{\left[e^{e}, \infty\right)},
$$

then a lengthy but straight-forward calculation shows that on $\left[e^{e^{e}}, \infty\right)$,

$$
M^{2} u(x) \approx x^{-1} \log x \log \log \log x .
$$

Let

$$
f(x)=(\log x \log \log x)^{-1} \chi_{\left[e^{e^{e}}, \infty\right)} ;
$$

then

$$
\int_{\mathbb{R}^{n}}|f|^{2} M_{L(\log L)} u d x=\int_{e^{e}}^{\infty} \frac{\log \log \log x}{x \log x(\log \log x)^{2}} d x<\infty .
$$

On the other hand, for $x>e^{e}, H f(x)=-\infty$, so

$$
u(\{x \in \mathbb{R}:|H f(x)|>1\}) \geq \int_{e^{e}}^{\infty} u d x=\infty .
$$

Therefore, inequality (1.5) does not hold.

Final Remark: Our proof of Theorem 1.2 yields a somewhat more general result. Theorem 3.1 clearly has weaker hypotheses, and though not so stated, the proof of inequality (1.6) in [16] shows that it holds for larger class of Young functions. If we combine hypotheses we get the following result whose details are left to the reader.

Theorem 4.1. Let $T$ be a Calderón-Zygmund operator. Given $p, 1<p<\infty$, suppose $A, B$, and $C$ are Young functions such that

(1) for any $t>0, B^{-1}(t) C^{-1}(t) \leq A^{-1}(t)$; 
(2) $A$ is doubling and there exists $q>1$ such that

$$
\int_{c}^{\infty}\left(\frac{t}{A(t)}\right)^{q-1} \frac{d t}{t}<\infty
$$

(3) $C$ is doubling and satisfies the $B_{p^{\prime}}$ condition.

Then, if $(u, v)$ is a pair of weights such that for every cube $Q$,

$$
\left\|u^{1 / p}\right\|_{B, Q}\left(\frac{1}{|Q|} \int_{Q} v^{-p^{\prime} / p} d x\right)^{1 / p^{\prime}} \leq K<\infty,
$$

inequality (1.5) holds for all $f \in L^{p}(v)$.

\section{References}

[1] C. Bennett and R. Sharpley, Interpolation of operators, Pure and Applied Mathematics, 129, Academic Press, Inc., Boston, MA, 1988.

[2] M. Carozza and A. Passarelli di Napoli, Composition of maximal operators, Publ. Mat. 40 (1996), 397-409.

[3] M. Christ, Lectures on singular integral operators, CBMS Regional Conference Series in Mathematics, 77, Amer. Math. Soc., Providence, R.I., 1990.

[4] R. Coifman and Y. Meyer, Au delà des opérateurs pseudo-différentiels, Astérisque $\mathbf{5 7}$ (1979), 1-84.

[5] D. Cruz-Uribe, SFO, and C. Pérez, Two-weight, weak-type norm inequalities for fractional integrals, Calderón-Zygmund operators and commutators, preprint.

[6] C. Fefferman and E.M. Stein, Some maximal inequalities, Amer. J. Math. 93 (1971), 107-115.

[7] J. García-Cuerva and J.L. Rubio de Francia, Weighted norm inequalities and related topics, North Holland Math. Studies 116, North-Holland Publishing Co., Amsterdam-New York, 1985.

[8] R. Hunt, B. Muckenhoupt and R. Wheeden, Weighted norm inequalities for the conjugate function and Hilbert transform, Trans. Amer. Math. Soc. 176 (1973), 227-252.

[9] J.-L. Journé, Zygmund operators, pseudo-differential operators and the Cauchy integral of Calderón, Lecture Notes in Mathematics, 994, Springer-Verlag, New York, 1983.

[10] M.A. Krasnosel'skiı̆ and Ya.B. Rutickiı̌, Convex functions and Orlicz spaces, P. Noordhoff Ltd., Groningen, 1961.

[11] B. Muckenhoupt, private communication.

[12] B. Muckenhoupt and R. Wheeden, Two weight function norm inequalities for the HardyLittlewood maximal function and the Hilbert transform, Studia Math. 60 (1976), 279-294.

[13] C.J. Neugebauer, Inserting $A_{p}$-weights, Proc. Amer. Math. Soc. 87 (1983), 644-648.

[14] R. O'Neil, Fractional integration in Orlicz spaces, Trans. Amer. Math. Soc. 115 (1965), 300-328.

[15] - Integral transforms and tensor products on Orlicz spaces and $L_{p, q}$ spaces, J. Analyse Math. 21 (1968), 1-276.

[16] C. Pérez, Weighted norm inequalities for singular integral operators, J. London Math. Soc. 49 (1994), 296-308.

[17] _ Two weighted inequalities for potential and fractional type maximal operators, Indiana Univ. Math. J. 43 (1994), 663-683.

[18] _ On sufficient conditions for the boundedness of the Hardy-Littlewood maximal operator between weighted $L^{p}$-spaces with different weights, Proc. London Math. Soc. 71 (1995), 135-157. 
[19] M.M. Rao and Z.D. Ren, Theory of Orlicz spaces, Monographs and Textbooks in Pure and Applied Mathematics, 146, Marcel Dekker Inc., New York, 1991.

[20] E.M. Stein, Note on the class L logL, Studia Math. 32 (1969), 305-310.

[21] S. Treil, A. Volberg, and D. Zheng, Hilbert transform, Toeplitz operators and Hankel operators, and invariant $A_{\infty}$ weights, Rev. Mat. Iberoamericana 13 (1997), 319-360.

Dept. of Mathematics, Trinity College, Hartford, CT 06106-3100, USA

E-mail address: david.cruzuribe@mail.trincoll.edu

Dep. de Matemáticas, Universidad Autónoma de Madrid, 28049 Madrid, Spain

E-mail address: carlos.perez@uam.es 